\title{
Characteristics of CSF Velocity-Time Profile in Posttraumatic Syringomyelia
}

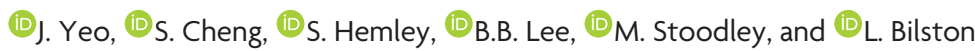

\begin{abstract}
BACKGROUND AND PURPOSE: The development of syringomyelia has been associated with changes in CSF flow dynamics in the spinal subarachnoid space. However, differences in CSF flow velocity between patients with posttraumatic syringomyelia and healthy participants remains unclear. The aim of this work was to define differences in CSF flow above and below a syrinx in participants with posttraumatic syringomyelia and compare the CSF flow with that in healthy controls.
\end{abstract}

MATERIALS AND METHODS: Six participants with posttraumatic syringomyelia were recruited for this study. Phase-contrast MR imaging was used to measure CSF flow velocity at the base of the skull and above and below the syrinx. Velocity magnitudes and temporal features of the CSF velocity profile were compared with those in healthy controls.

RESULTS: CSF flow velocity in the spinal subarachnoid space of participants with syringomyelia was similar at different locations despite differences in syrinx size and locations. Peak cranial and caudal velocities above and below the syrinx were not significantly different (peak cranial velocity, $P=.9$; peak caudal velocity, $P=1.0)$, but the peak velocities were significantly lower $(P<.001, P=.007)$ in the participants with syringomyelia compared with matched controls. Most notably, the duration of caudal flow was significantly shorter $(P=.003)$ in the participants with syringomyelia.

CONCLUSIONS: CSF flow within the posttraumatic syringomyelia group was relatively uniform along the spinal canal, but there are differences in the timing of CSF flow compared with that in matched healthy controls. This finding supports the hypothesis that syrinx development may be associated with temporal changes in spinal CSF flow.

S yringomyelia is a neurologic condition characterized by the development of a syrinx, a fluid cyst in the spinal cord. It is commonly associated with conditions that obstruct spinal CSF flow such as spinal cord injury, ${ }^{1}$ Chiari type I malformation, and spinal tumors. Syrinxes form and enlarge in either the central canal of the spinal cord or in the cord parenchyma. For a syrinx to enlarge, the laws of mechanics require that the syrinx pressure

Received February 20, 2017; accepted after revision April 24

From Neuroscience Research Australia (J.Y., B.B.L., L.B.), Randwick, New South Wales, Australia; Department of Engineering (S.C.), Faculty of Science and Engineering, and Australian School of Advance Medicine (S.H., M.S.), Macquarie University, Sydney, New South Wales, Australia; Prince of Wales Hospital (B.B.L.), Sydney, New South Wales, Australia; and Prince of Wales Clinical School (B.B.L., L.B.), University of New South Wales, Kensington, New South Wales, Australia.

This research was supported by the Australian National Health and Medical Research Council (APP1063628). L.B. is supported by National Health and Medical Research fellowship. The authors would also like to acknowledge the Column of Hope for funding part of this project.

Please address correspondence to Shaokoon Cheng, PhD, Department of Engineering, Faculty of Science and Engineering, Balaclava Rd, North Ryde, NSW, 2109, Australia; e-mail: shaokoon.cheng@mq.edu.au

-- Indicates open access to non-subscribers at www.ajnr.org

http://dx.doi.org/10.3174/ajnr.A5304 exceed the pressure in the surrounding cord tissue and spinal subarachnoid space. However, the mechanism of CSF flow into a syrinx in the presence of this reverse pressure gradient is poorly understood and remains controversial. Computational models suggest that CSF could be driven by cardiac pulsations from the spinal subarachnoid space into the spinal cord via periarterial spaces, including toward a syrinx. ${ }^{2}$ Besides CSF, another possible source of syrinx fluid could be extracellular fluid. It has recently been shown that after spinal cord injury, the blood-spinal cord barrier is damaged for an extended time ${ }^{3}$ and fluid could hence pass from the vasculature into a syrinx. However, the source of fluid in the syrinx has yet to be identified because the chemical composition of CSF and extracellular fluid is indistinguishable. ${ }^{4}$

Understanding the characteristics of CSF dynamics in the spinal subarachnoid space and the way they change in conditions associated with syringomyelia may help elucidate the mechanism of the disease. Characterizing CSF flow in syringomyelia may also improve clinical management because syrinx morphology from MR anatomic images alone is insufficient to predict disease progression and surgical outcomes. Current treatment techniques for posttraumatic syringomyelia, such as shunting, are associated 
with syrinx recurrence. Therefore, understanding the CSF flow characteristics in these patients may help in developing effective techniques to manage this complex condition.

CSF flow in the spinal subarachnoid space consists of pulsatile caudal and rostral flow during systole and diastole, respectively. ${ }^{5}$ Caudal flow in the spinal subarachnoid space commences approximately $100 \mathrm{~ms}$ after the onset of systole in healthy individuals, and the timing of its onset is affected by age and CSF obstructions in the spinal subarachnoid space. Detailed mechanisms that underpin the earlier onset of peak caudal CSF are not yet wellestablished and may be influenced by compliance in the craniospinal system. In the spinal subarachnoid space of healthy individuals, peak caudal and cranial velocities and their onset vary with spinal level. However, these variables are different in those with Chiari malformation. ${ }^{6}$

Despite numerous studies in the literature of CSF flow in participants with Chiari type I malformation with and without syrinxes, there is a lack of understanding of spinal CSF dynamics in those who have sustained a spinal cord injury. Therefore, this study aimed to determine the CSF velocity-time profiles adjacent to the syrinx in participants with spinal cord injury and compare them with those in healthy controls. It is hypothesized that the peak CSF velocities and timing of the profile would be significantly altered in patients with posttraumatic syringomyelia.

\section{MATERIALS AND METHODS \\ Participants}

This study was approved by the South Eastern Sydney Local Health District Human Research Ethics Committee (Sydney, New South Wales, Australia). Participants were recruited from the spinal injury outpatient clinic at the Prince of Wales Hospital, Sydney, New South Wales, Australia. All patients had an American Spinal Injury Association (ASIA) impairment scale score of A except for 1 participant with no recorded ASIA score. ${ }^{7}$ None had past spinal surgery. They were informed about the details of the study on at least 2 separate occasions before their scan, and written informed consent was obtained. They were also carefully screened for MR imaging safety. Six participants (4 men, 2 women; mean age, $46.5 \pm 13.9$ years) previously diagnosed with posttraumatic syringomyelia were selected for the study.

The participants with syringomyelia were recruited prospectively. Their data were compared with retrospectively reanalyzed scanned data of 6 healthy controls ( 3 men, 3 women) matched for age (mean age, $45 \pm 18.1$ years) and weight. On the basis of a statistical power analysis, a sample size of 6 was determined to adequately demonstrate significant differences in the characteristics of the CSF velocity-time profile between the participants with syringomyelia and healthy controls based on a mean difference of $0.15 \pm 0.09 \mathrm{~cm} / \mathrm{s}$ and $80 \%$ power in a 2-tailed $t$ test.

\section{Imaging Protocols}

MR imaging measurements were performed on a 3T MR imaging scanner (Achieva TX; Philips Healthcare, Best, the Netherlands). Depending on the syrinx location, participants were scanned with a neurovascular coil and/or a 32-channel thoracic coil. Meanwhile, the healthy controls were retrospectively scanned with a neurovascular coil. All participants were scanned without any an-

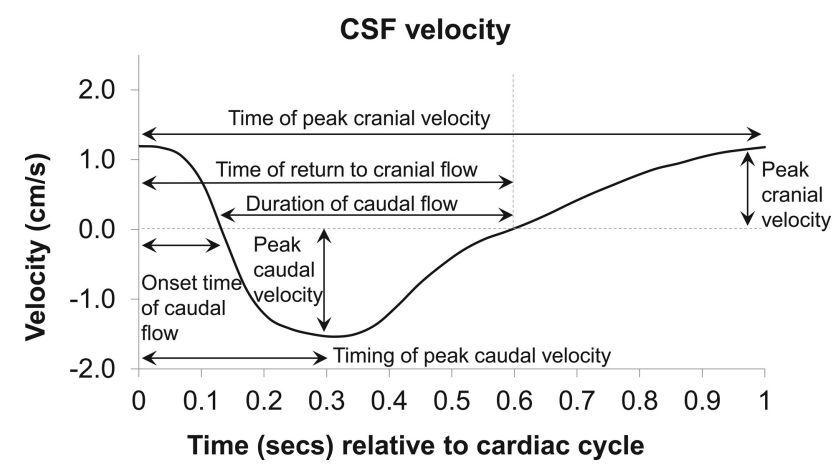

FIG 1. Representative CSF velocity profile from a healthy control volunteer showing measurements collected for analysis.

esthesia. A T1-weighted sequence was performed in the midsagittal plane to verify the syrinx location. The parameters of the sequence were the following: flip angle $=10^{\circ}, \mathrm{FOV}=200 \times 200$ $\mathrm{mm}, \mathrm{TR} / \mathrm{TE}=21 / 7.3 \mathrm{~ms}$, and section thickness $=5 \mathrm{~mm}$. Phasecontrast MR imaging was used to measure CSF flow velocity, and cardiac gating was achieved with vector cardiogram leads. Thirty cardiac phase images were obtained from the onset of the $\mathrm{R}$ wave. CSF flow velocities at the base of the skull and above and below the syrinx spinal level were measured with an axial imaging plane aligned perpendicular to the spinal cord. The encoding velocities for the scans were $10 \mathrm{~cm} / \mathrm{s}$ for the base of the skull and $12-14 \mathrm{~cm} / \mathrm{s}$ for other spinal levels. Control flows were measured at the base of skull and the C5 spinal level. The imaging parameters for this sequence were the following: flip angle $=$ $10^{\circ}$, matrix $=240 \times 176, \mathrm{FOV}=250 \times 250 \mathrm{~mm}, \mathrm{TR}=21 \mathrm{~ms}$, $\mathrm{TE}=6.8 \mathrm{~ms}$, and section thickness $=5 \mathrm{~mm}$. The duration of each scan session, including setup and subject positioning, varied among participants and ranged from 1 to 1.5 hours. The heart rates of the participants with syrinx and the controls during the scan were $64.3 \pm 4.6$ beats per minute and $69.0 \pm$ 15.0 beats per minute, respectively, and they were not significantly different $(P=.4)$.

\section{Data Analysis}

CSF velocity-time profiles were measured from phase-contrast MR images with Segment. ${ }^{8}$ ROIs were defined (2 lateral, 1 anterior, 1 posterior) with circular monitoring points of approximately $2 \mathrm{~mm}$ in diameter. The average CSF velocity-time profiles of the 4 ROIs were obtained at the 3 spinal levels (base of the skull and above and below the syrinx), and features of the profile were analyzed with the same protocol as that of Clarke et al. ${ }^{9}$ The features measured were maximum velocities in the cranial and caudal direction and their timing, onset time and duration of caudal flow, and time of return to cranial flow. A representative CSF velocity-time profile from a healthy control is shown in Fig 1.

Features of the velocity-time profiles were compared for the following: 1) above and below the syrinx in participants with syringomyelia, 2) the base of the skull in participants with syringomyelia and healthy controls, and 3) the spinal level near the syrinx in participants with syringomyelia (between $\mathrm{C} 4$ and T12) and healthy controls (C5). A paired $t$ test was used to analyze differences in CSF flow above and below the syrinx, and an unpaired $t$ 
test was used for all other statistical analyses. A Bonferroni post hoc correction was performed by adjusting the significance level of the $t$ tests from .05 to .007 . This was obtained by dividing .05 by 7 because there were 7 hypotheses (representing the 7 features of the CSF velocity-time profile) that would be tested using the average velocity-time profile.

Table 1: Syrinx maximum transverse diameter and location in participants $^{\mathrm{a}}$

\begin{tabular}{|c|c|c|c|c|}
\hline $\begin{array}{l}\text { Patients } \\
\text { with } \\
\text { Syrinx }\end{array}$ & $\begin{array}{c}\text { Syrinx } \\
\text { Location }\end{array}$ & $\begin{array}{l}\text { Spinal } \\
\text { Injury }\end{array}$ & $\begin{array}{l}\text { Maximum } \\
\text { Syrinx } \\
\text { Diameter } \\
(\mathrm{mm})\end{array}$ & Flow Measurements \\
\hline SPI & T6-T7 & T6 & 4.2 & $\mathrm{BOS}, \mathrm{T}^{\mathrm{b}}(\mathrm{AS}), \mathrm{T}$ (BS) \\
\hline SP2 & $\mathrm{C} 6-\mathrm{Tl}$ & C6 & 17.1 & $\mathrm{BOS}, \mathrm{C} 4^{\mathrm{b}}(\mathrm{AS}), \mathrm{T} 2(\mathrm{BS})$ \\
\hline SP3 & $\mathrm{Cl}-\mathrm{C} 6$ & C6 & 23.4 & $\mathrm{BOS}(\mathrm{AS}), \mathrm{C} 4{ }^{\mathrm{b}} \mathrm{Tl}(\mathrm{BS})$ \\
\hline SP4 & $\mathrm{T} 2-\mathrm{T} 6$ & T5 & 8.2 & $\mathrm{BOS}, \mathrm{T}^{\mathrm{b}}(\mathrm{AS}), \mathrm{T}(\mathrm{BS})$ \\
\hline SP5 & $\mathrm{Tl}-\mathrm{T} 4$ & T3 & 15.0 & $\mathrm{C}^{\mathrm{b}}(\mathrm{AS}), \mathrm{T} 5$ (BS) \\
\hline SP6 & L1-L2 & L1 & 9.2 & $\mathrm{BOS}, \mathrm{T} 12^{\mathrm{b}}(\mathrm{AS}), \mathrm{L} 2$ (BS) \\
\hline
\end{tabular}

Note:-AS indicates above the syrinx; BS, below the syrinx; BOS, base of skull.

${ }^{a}$ Data obtained at these levels were used to compare flow in the participants with syrinx (Fig 2).

${ }^{\mathrm{b}}$ CSF flow measurements obtained at this spinal level were used to compare flow with that of controls at C5 (Fig 3).

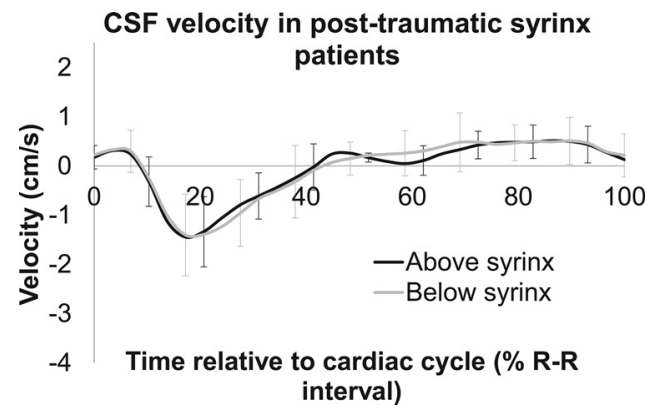

FIG 2. Mean CSF velocity-time profiles in participants with syrinx for above and below the syrinx. SDs are represented by error bars.

Table 2: Summary of features of CSF velocity-time profile above and below the syrin $x^{a}$

\begin{tabular}{llcc}
\hline & $\begin{array}{c}\text { Above } \\
\text { Syrinx }\end{array}$ & $\begin{array}{c}\text { Below } \\
\text { Syrinx }\end{array}$ & $\begin{array}{c}\boldsymbol{P} \\
\text { Value }\end{array}$ \\
\hline Timing of peak cranial velocity & $8.8(2.5)$ & $9.0(1.8)$ & .9 \\
Timing of peak caudal velocity & $2.3(1.1)$ & $2.3(0.7)$ & 1.0 \\
Duration of caudal flow & $3.5(0.5)$ & $4.3(2.5)$ & .5 \\
Onset time of caudal flow & $1.0(0.2)$ & $1.1(0.5)$ & .6 \\
Time of return to cranial flow & $4.6(0.6)$ & $5.5(2.2)$ & .3 \\
Peak cranial velocity (cm/s) & $0.6(0.3)$ & $0.7(0.40)$ & .7 \\
Peak caudal velocity $(\mathrm{cm} / \mathrm{s})$ & $1.6(0.9)$ & $1.7(0.9)$ & .7 \\
\hline
\end{tabular}

${ }^{a}$ Data are mean (SD). All temporal features are expressed as \% R-R interval.

CSF velocity (Base of skull)

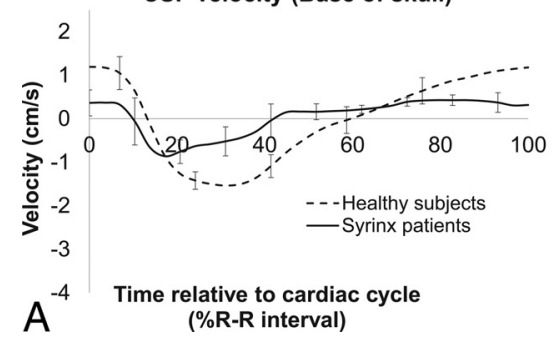

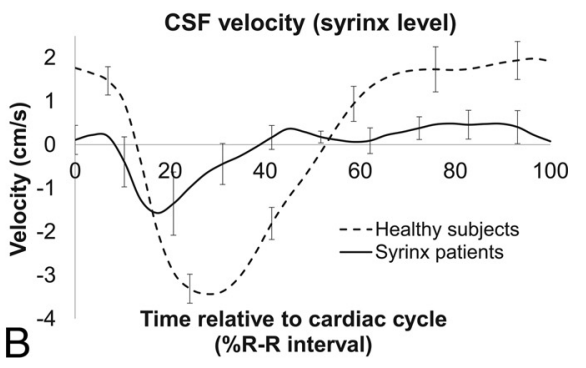

FIG 3. Average CSF velocity-time profile in participants with syrinx (black line) and controls (dotted line) at the skull base $(A)$ and near the syrinx $(B)$ (see Table 1 for locations of measurements in participants with syrinx) and at the C5 spinal level in controls. Error bars represent SDs.

\section{RESULTS}

Syrinxes were observed in the T1-weighted MR images in all participants with syringomyelia. Syrinx size varied among participants. Table 1 summarizes the location of the syrinx and spinal levels where flow measurements were obtained. CSF flow velocity at the base of the skull was not measured for participant SP5 because of an existing irregular heartbeat, which made cardiac gating difficult. The scan was terminated due to prolonged scan time and patient discomfort. In addition, CSF velocity was measured 2 spinal levels (C4) above the syrinx for participant SP2 because the immediate spinal level (C5) above the syrinx could not be visualized because the spinal cord was obscured by spinal injury.

\section{CSF Velocity in Participants with Syrinx}

Figure 2 shows the average CSF flow velocity above and below the syrinxes. Despite differences in syrinx size, location, and spinal subarachnoid space geometry in the participants with syringomyelia, there was very little difference in CSF flow velocity above and below the syrinx, and this is demonstrated by the small SDs in Fig 2. In addition, there were no significant differences in any features of the CSF velocity-time profiles above and below the syrinx (paired $t$ tests, all $P>.1$, see Table 2 for details). In general, CSF flow was initially cranially directed (positive velocity); then, it was reversed to caudal flow at $\sim 10 \%$ of the cardiac cycle and then reversed again to cranial flow at $\sim 40 \%$ of the cardiac cycle. There was little variation in this timing along the spinal canal in the patients with syrinx (Table 2). The peak caudal and cranial velocities above and below the syrinx were similar despite the differences in syrinx location along the spine. In addition, in the participants with syrinx, the CSF flow velocity at the base of the skull (Fig $3 \mathrm{~A}$, black line) was also similar to the CSF flow velocity above and below the syrinx.

\section{CSF Velocity in Patients with Syringomyelia and Healthy Participants}

Figure $3 A$ shows the comparison between CSF flow velocity at the base of the skull in the controls and the participants with posttraumatic syringomyelia. Although the onset time of caudal flow appeared to be $\sim 3 \%$ earlier in the participants with syringomyelia than in the controls, this was not significantly different $(P=.15)$. However, the average peak cranial and caudal velocities in the participants with syringomyelia were $46 \%$ and $65 \%$ lower than those in controls, respectively, and these were significantly different between groups (peak cranial velocity, $P=.01$; peak caudal velocity, $P=.03$ ).

The average CSF flow-velocity profiles obtained at the syrinx level (spinal levels between $\mathrm{C} 4$ and T12) in the participants with syringomyelia and at spinal level C5 in the controls are shown in Fig $3 B$. All the features of the CSF velocity-time profile at this level were significantly different between patients and controls (see Table 3 for details) except for the timing of peak cranial velocity $(8.8 \pm 2.5$ seconds for participants with syringomyelia, $8.3 \pm 4.5$ seconds in con- 
Table 3: Summary of features of CSF velocity time-profile in participants with syrinx and controls at the base of the skull and syrinx spinal level ${ }^{\mathrm{a}}$

\begin{tabular}{|c|c|c|c|c|c|c|}
\hline & \multicolumn{3}{|c|}{ Base of Skull } & \multicolumn{3}{|c|}{ Syrinx Spinal Level } \\
\hline & Patients with Syrin $x$ & Controls & $P$ Value & Patients with Syrinx & Controls & $P$ Value \\
\hline Timing of peak cranial velocity & $8.8(2.3)$ & $4.2(5.2)$ & .21 & $8.8(2.5)$ & $8.3(4.5)$ & .4 \\
\hline Timing of peak caudal velocity & $2.3(0.9)$ & $3.7(1.2)$ & .07 & $2.3(1.1)$ & $3.6(0.7)$ & .03 \\
\hline Duration of caudal flow & $3.5(0.9)$ & $5.4(0.5)$ & .05 & $3.5(0.5)$ & $4.9(0.6)$ & .003 \\
\hline Onset time of caudal flow & $1.3(0.5)$ & $1.6(0.4)$ & .15 & $1.0(0.2)$ & $1.7(0.4)$ & .002 \\
\hline Time of return to cranial flow & $4.8(1.3)$ & $7(0.9)$ & .04 & $4.6(0.6)$ & $6.6(0.9)$ & .002 \\
\hline Peak cranial velocity $(\mathrm{cm} / \mathrm{s})$ & $0.6(0.2)$ & $1.3(0.3)$ & .01 & $0.6(0.3)$ & $2.0(0.5)$ & .00004 \\
\hline Peak caudal velocity $(\mathrm{cm} / \mathrm{s})$ & $1.1(0.2)$ & $1.7(0.2)$ & .03 & $1.6(0.9)$ & $3.2(0.7)$ & .007 \\
\hline
\end{tabular}

${ }^{a}$ Data are mean (SD). All temporal features are expressed as \% R-R interval. $P$ values are from unpaired $t$ tests between participants with syrinx and controls.

trols, unpaired $t$ test, $P=.4$ ). The average peak cranial and caudal velocities in the controls were 3.3 and 2 times higher, respectively, than those in the participants with syringomyelia. The CSF flow profile relative to the cardiac cycle was shifted earlier in the participants with syrinx relative to the controls (Fig $3 B$ ).

\section{DISCUSSION}

There were 2 key observations in this novel study of CSF flow profiles in patients with posttraumatic syringomyelia. First, all the features of the CSF velocity-time profile were significantly different between the participants with syringomyelia and matched controls, with most flow features occurring earlier in the cardiac cycle in participants with syringomyelia than in healthy controls. In addition, while there were obvious differences in CSF flow velocity at different spinal levels (base of the skull and C5) in healthy controls, CSF flow along the spine was more uniform in the participants with syringomyelia.

Results from this study support the hypothesis that syrinxes are associated with altered CSF flow. This study shows that the timing of the peak caudal velocity in the participants with syringomyelia was earlier by $3 \%-5 \%$ of the cardiac cycle than in matched controls. This difference in timing of the CSF flow implies that there will also be an earlier arrival of peak spinal CSF pressure relative to cardiac systole in the participants with syringomyelia. ${ }^{10}$ A modest change in the time of arrival of peak CSF pressure relative to the arrival of peak arterial pressure in systole may enhance CSF flow into the spinal cord through the perivascular spaces, ${ }^{11,12}$ though this possibility remains to be confirmed experimentally. Syringomyelia is associated with CSF flow obstructions. CSF flow obstructions in Chiari malformation, ${ }^{10,13}$ spinal stenosis, and arachnoiditis ${ }^{14}$ are associated with increased velocity at the level of obstruction and a large pressure drop across the obstruction on the basis of existing computational modeling studies on CSF flow in the spinal subarachnoid space. According to existing hypotheses, syrinxes occur due to the development of pressure differences along the spinal subarachnoid space. This pressure difference could either drive CSF into the spinal cord ${ }^{15}$ or distend the spinal cord radially due to Venturi effects. ${ }^{16}$

Most interesting, this study shows that both the peak caudal and cranial CSF velocities above and below the syrinx were similar; therefore, any CSF pressure drop across the syrinx in the spinal subarachnoid space would be small. In fact, the CSF pressure drop along the spinal subarachnoid space would be higher in the healthy controls because of a significant change in CSF flow velocity. This can be observed in the average peak caudal velocity, where the velocity at C5 was almost 2 times higher compared with that in the base of the skull. It is therefore tempting to believe that the CSF pressure drop across a syrinx has little to do with the pathogenesis of syringomyelia, at least in posttraumatic participants. Longitudinal monitoring of patients after spinal cord injury would be required to confirm this belief by capturing any differences in CSF flow before the formation of a syrinx and during syrinx enlargement. However, this was not possible in this cross-sectional study design. Furthermore, this study was unable to investigate specific changes in the temporal features of CSF flow as a syrinx enlarges.

Nevertheless, although there were minimal differences in CSF velocity (and therefore pressure) above and below the syrinx, the absolute CSF pressure in the participants with syringomyelia may be higher than in controls because the disease is known to be related to decreased craniospinal compliance. ${ }^{17}$ The decrease in craniospinal compliance may increase the effects of arterial pulsations, which may increase perivascular flow into the spinal cord parenchyma and syrinx.

Most interesting, alterations in the CSF flow profile in patients with posttraumatic syringomyelia differed from those in studies that compared patients with Chiari type I malformation with matched controls. ${ }^{9}$ In patients with posttraumatic syringomyelia, peak caudal velocity occurred earlier in the cardiac cycle than in matched controls. Conversely, there was no significant difference in the timing of peak caudal velocity between patients with Chiari malformation and matched controls. Nevertheless, several temporal features of the CSF velocity profiles being different between patients with Chiari malformation with and without a syrinx suggests that the temporal effects of CSF flow velocity are still likely to play a role in syrinx development. In patients with Chiari malformation without a syrinx, the onset of caudal flow, return to cranial flow, and peak caudal velocity all occurred significantly earlier in the cardiac cycle compared with patients with a syrinx. It is unclear whether treatment can effectively reverse the temporal changes in CSF flow dynamics in syringomyelia, but there are at least 2 studies that showed that treatment of CSF flow disorders could delay the onset time of caudal flow and peak caudal velocity. A study that investigated CSF flow velocity in patients with Chiari malformation (after a decompression operation) showed that the duration of caudal CSF flow appeared to be shorter than that in flow measured postoperatively. ${ }^{18}$ Temporal changes in CSF flow may also be associated with other CSF disorders, such as hydrocephalus. A computational modeling study of hydrocephalus found that the peak caudal velocity occurred later in the cardiac 
cycle after a simulated endoscopic third ventriculostomy at the third ventricle. ${ }^{19}$

There were several limitations to this study. First, the primary motivation of this research was to understand CSF flow velocity above and below the syrinx in posttraumatic patients. However, there was a discrepancy in the comparison of spinal levels between participants with syringomyelia and healthy controls in some cases. Although the average CSF flow velocity obtained adjacent to the syrinx varied between $\mathrm{C} 4$ and $\mathrm{T} 12$ in our participants, it was compared with CSF flow velocity at a standard spinal location (C5) in the matched controls. Nevertheless, given that there was minimal difference in CSF flow velocities along the spine in the patients with syrinx, we expected the effect of these differences in measurement locations to be small. Comparing the magnitude of CSF velocity measurements in the participants with syringomyelia with that in the healthy controls at a different spinal location (eg, T1) may yield different results because CSF velocity is highest at the $\mathrm{C} 5$ level in healthy humans and can be reduced by as much as $20 \%-30 \%$ at the thoracic spinal region. ${ }^{20}$ However, when CSF velocity in the participants with syringomyelia was compared with a simulated CSF velocity-time profile that has peak velocity reduced by $20 \%$, the results were still significantly different for both the cranial and caudal velocities.

Second, it was not anticipated before this study that the CSF velocity of participants with posttraumatic syringomyelia would be in the range of $1-3 \mathrm{~cm} / \mathrm{s}$, and because this was only identified as a consistent observation midway in the study, the encoding velocity was left unchanged to ensure consistencies in the protocol used. While this feature may affect the measurement accuracy of the velocity magnitude, it is unlikely to change the conclusions of this study.

Another limitation was that data were not collected from spinal patients without a syrinx for comparison. Because this was a cross-sectional study and neither prior nor subsequent measurements of syrinx dimensions were available from the participants' clinical records, we are unable to comment on whether syrinxes in the participants were stable. Understanding longitudinal changes in CSF flow measurements and syrinx size in the participants with syringomyelia may help provide insights into the mechanisms of syrinx development, but this was not designed as part of this study. It would also be ideal to compare the CSF velocity-time profile with spinal arterial blood flow to assess the theory that change in the relative timing of arterial blood and CSF pulses is important for syrinx development and progression. ${ }^{2}$ However, neither arterial blood flow nor additional spinal level scans were measured in this study because patient discomfort precluded additional scan acquisitions. Finally, the sample size of this study, though small, was appropriate, and while a lager sample size will provide a more robust conclusion to the study, it is unlikely to affect the key findings.

\section{CONCLUSIONS}

This study provides new information on CSF flow profiles in patients with a syrinx with spinal cord injuries. In the participants with syringomyelia, there was very little difference in peak caudal and cranial velocities above and below the syrinx. This suggests that there was an absence of a large pressure drop across the syrinx. However, peak CSF caudal and cranial velocities in the par- ticipants with syringomyelia were significantly lower compared with those in the healthy controls. Furthermore, temporal features of the CSF flow profile were significantly different between the 2 groups; peak flow and change in flow direction were earlier in the cardiac cycle in participants with syringomyelia. Additional work to study the specific changes in the temporal features of CSF flow when a syrinx enlarges and posttreatment is important to further elucidate the mechanisms of syringomyelia development. This work could assist in predicting treatment response.

\section{ACKNOWLEDGMENTS}

The authors thank Mr Segar Suppiah from Neuroscience Research Australia for his help with MR imaging.

Disclosures: Shaokoon Cheng—RELATED: Grant: Column of Hope. Marcus Stoodley-RELATED: Grant: National Health and Medical Research Council, Column of Hope, Comments: standard competitive research grants*. Lynne Bilston-RELATED: Grant: National Health and Medical Research Council of Australia* UNRELATED: Grants/Grants Pending: National Health and Medical Research Council, Australian Research Council, Column of Hope, University of New South Wales, Rebecca Cooper Foundation*. *Money paid to the institution.

\section{REFERENCES}

1. Brodbelt AR, Stoodley MA. Post-traumatic syringomyelia: a review. J Clin Neurosci 2003;10:401-08 CrossRef Medline

2. Bilston LE, Fletcher DF, Brodbelt AR, et al. Arterial pulsation-driven cerebrospinal fluid flow in the perivascular space: a computational model. Comput Methods Biomech Biomed Engin 2003;6:235-41 CrossRef Medline

3. Hemley SJ, Tu J, Stoodley MA, et al. Role of the blood-spinal cord barrier in posttraumatic syringomyelia. J Neurosurg Spine 2009;11: 696-704 CrossRef Medline

4. Naruse H, Tanaka K, Kim A, et al. A new model of spinal cord edema. Acta Neurochir Suppl 1997;70:293-95 Medline

5. Enzmann DR, Pelc NJ. Normal flow patterns of intracranial and spinal cerebrospinal fluid defined with phase-contrast cine MR imaging. Radiology 1991;178:467-74 CrossRef Medline

6. Mauer UM, Gottschalk A, Mueller C, et al. Standard and cardiacgated phase-contrast magnetic resonance imaging in the clinical course of patients with Chiari malformation type I. Neurosurg Focus 2011;31:E5 CrossRef Medline

7. Maynard FM, Bracken MB, Creasey G, et al. International Standards for Neurological and Functional Classification of Spinal Cord Injury: American Spinal Injury Association. Spinal Cord 1997;35: 266-74 CrossRef Medline

8. Heiberg E, Sjögren J, Ugander M, et al. Design and validation of Segment: freely available software for cardiovascular image analysis. BMC Med Imaging 2010;10:1 CrossRef Medline

9. Clarke EC, Stoodley MA, Bilston LE. Changes in temporal flow characteristics of CSF in Chiari malformation type I with and without syringomyelia: implications for theory of syrinx development. J Neurosurg 2013;118:1135-40 CrossRef Medline

10. Clarke EC, Fletcher DF, Stoodley MA, et al. Computational fluid dynamics modelling of cerebrospinal fluid pressure in Chiari malformation and syringomyelia. J Biomech 2013;46:1801-09 CrossRef Medline

11. Bilston LE, Stoodley MA, Fletcher DF. The influence of the relative timing of arterial and subarachnoid space pulse waves on spinal perivascular cerebrospinal fluid flow as a possible factor in syrinx development. J Neurosurg 2010;112:808-13 CrossRef Medline

12. Stoodley MA, Brown SA, Brown CJ, et al. Arterial pulsation-dependent perivascular cerebrospinal fluid flow into the central canal in the sheep spinal cord. J Neurosurg 1997;86:686-93 CrossRef Medline

13. Martin BA, Kalata W, Shaffer N, et al. Hydrodynamic and longitudinal impedance analysis of cerebrospinal fluid dynamics at the 
craniovertebral junction in type I Chiari malformation. PLoS One 2013;8:e75335 CrossRef Medline

14. Cheng S, Stoodley MA, Wong J, et al. The presence of arachnoiditis affects the characteristics of CSF flow in the spinal subarachnoid space: a modelling study. J Biomech 2012;45:1186-91 CrossRef Medline

15. Williams B. On the pathogenesis of syringomyelia: a review. J R Soc Med 1980;73:798-806 Medline

16. Greitz D. Unraveling the riddle of syringomyelia. Neuros Rev 2006; 29:251-63; discussion 264 CrossRef Medline

17. Rusbridge C, Greitz D, Iskandar BJ. Syringomyelia: current concepts in pathogenesis, diagnosis, and treatment. J Vet Intern Med 2006;20: 469-79 CrossRef Medline
18. Sivaramakrishnan A, Alperin N, Surapaneni S, et al. Evaluating the effect of decompression surgery on cerebrospinal fluid flow and intracranial compliance in patients with Chiari malformation with magnetic resonance imaging flow studies. Neurosurgery 2004;55: 1344-50; discussion 1350-51 CrossRef Medline

19. Farnoush A, Tan K, Juge L, et al. Effect of endoscopic third ventriculostomy on cerebrospinal fluid pressure in the cerebral ventricles. J Clin Neurosci. 2016;23:63-67 CrossRef Medline

20. Heidari Pahlavian S, Bunck AC, Loth F, et al. Characterization of the discrepancies between four-dimensional phase-contrast magnetic resonance imaging and in-silico simulations of cerebrospinal fluid dynamics. J Biomech Eng 2015;137:051002 CrossRef Medline 